\title{
CONDICIÓN DE SALUD Y CUMPLIMIENTO DE LA GUÍA CLÍNICA CHILENA PARA EL CUIDADO DEL PACIENTE DIABÉTICO TIPO $2^{1}$
}

\author{
HEALTH CONDITION AND COMPLIANCE OF THE CHILEAN \\ CLINICAL GUIDELINE FOR TYPE 2 DIABETIC PATIENTS
}

\author{
Ma Alejandra Galiano G. \\ Ma Silvia Calvo A.** \\ Ma Alicia Feito T.** \\ Ma Waleska Aliaga B. ${ }^{* * *}$ \\ SARA LEIVA M. ${ }^{* * * *}$ \\ BeATriz Mujica P. ${ }^{* * * * *}$
}

\begin{abstract}
RESUMEN
Objetivo: Identificar condición de salud (CS) de los pacientes diabéticos tipo 2. Medir el cumplimiento de las atenciones de salud según guía clínica. Identificar variables asociadas a CS. Material y método: estudio de prevalencia, abril 2010. Población: 1.100 pacientes DM2 controlados en Programa Cardiovascular, considerando criterios de inclusión, exclusión, consentimiento informado. Muestra: 340 por aleatorización simple (confianza 95\%, merma 10\%). Recolección de datos realizada en CESFAM o visita domiciliaria por investigadoras e internas de enfermería entrenadas, con encuesta validada, exámenes, plantilla para información y ficha clínica. Para el análisis se utilizó estadística descriptiva, Chi2, Odds Ratios con IC (95\%). Resultados: CS: descompensados (HbAlc $>7 \%$ ) 56.5\%, patologías agregadas $97.9 \%$, complicaciones $25 \%$, mayor frecuencia retinopatía diabética. Hombres mayor riesgo de amputación ( $\mathrm{p}=0.003$ ). Presión arterial $>130 / 85 \mathrm{~mm} . \mathrm{Hg}$. 58.2\%, sobrepeso-obesidad $71.2 \%$, autovalencia (adultos mayores) 23.1\%. Cumplimiento recomendaciones ministeriales: controles/profesionales/año a lo menos cinco 41.5\%, CESFAM adapta esta recomendación. Evaluación anual de: pie diabético $78 \%$, fondo ojo $41,6 \%$. Indicación régimen (nutricionista) último control $80 \%$. Cinco controles profesionales/ año o más es protector de compensación de diabetes, IC (0.62-0.95), resto de recomendaciones y adaptación de éstas no se asocian a condición de salud ( $\mathrm{p}>0.05)$. Conclusiones: Los componentes de condición de salud están en general por sobre lo esperado. En ningún paciente se cumple en un 100\% las recomendaciones de la guía clínica. Cinco o más controles profesionales anuales se asocian a compensación de la diabetes. Importante cumplir recomendaciones para mejorar control metabólico y disminuir/retrasar complicaciones para una mejor calidad de vida de pacientes.
\end{abstract}

Palabras clave: Diabetes mellitus tipo 2, guías de práctica clínica, atención de salud.

\footnotetext{
${ }^{1}$ Financiamiento: Servicio de Salud Metropolitano Oriente (SSMO), Santiago, Chile. Fondo de Ayuda a la Investigación (FAI), Universidad de los Andes. Santiago, Chile.

${ }^{*}$ Enfermera. Docente Universidad de los Andes. Santiago, Chile. Av. San Carlos de Apoquindo 2200, Las Condes. Santiago.

E-mail: magalianog@uandes.cl

${ }^{* *}$ Enfermera. Docente Universidad de los Andes. Santiago, Chile. E-mail: calsilvia@gmail.com

*** Enfermera. Docente Universidad de los Andes. Santiago, Chile. E-mail: mafeitotapia@gmail.com

${ }^{* * * *}$ Enfermera. CESFAM Félix de Amesti. Santiago, Chile. E-mail: maria_aliaga@vtr.net

${ }^{* * * * *}$ Enfermera. CESFAM Félix de Amesti. Santiago, Chile. E-mail: sleiva@corpomunimacul.cl

${ }^{* * * * * *}$ Estadístico. Docente Universidad de los Andes. Santiago, Chile. E-mail: bmujica@vtr.net
} 


\section{ABSTRACT}

Objectives: To determine compliance with Chilean clinical guideline and to measure their impact through evaluation of patient's health condition (HC), to determine associations. Methods: prevalence study, April 2010. Population: 1,100 diabetic patients controlled in the Cardiovascular Program, considering criteria of inclusion, exclusion, informed consent. Sample: 340 randomized patients (Confidence level: 95\%; Loss: 10\%). Information was collected in an outpatient clinic and through home visits performed by researchers and trained senior nursing students, with a validated survey, exams, staff for file information. For the purposes of the analysis, descriptive statistics was used, Chi2, Odds Ratios with CI (95\%). Results: HC: decompensation (HbAlc $>7 \%$ ) 56.5\%, derived illnesses $97.9 \%$, complications $25 \%$, greater frequency of diabetic retinopathy. Males with greater risk of amputation $(\mathrm{p}=0.003)$. Blood pressure $>130 / 85 \mathrm{~mm}$. Hg. $58.2 \%$, overweight-obesity $71.2 \%$, self-care (older adults) $23.1 \%$. Compliance with clinical guideline: controls/professionals/year at least five $41.5 \%$, CESFAM adapts this recommendation. Annual evaluation of: diabetic foot $78 \%$, fundoscopy $41.6 \%$. Regime indication (dietician) last control $80 \%$. Five professional controls/year or more protect compensation for diabetes, IC (0.62-0.95), other recommendations and adaptation of recommendations are not associated with health status $(\mathrm{p}>0.05)$. Conclusions: components of $\mathrm{HC}$ are better than expected. In none of the patients are the recommendations of the clinical guideline fulfilled in a $100 \% .5$ or more annual outpatient visits are associated to good metabolic control. Compliance with clinical guideline recommendations is important to improve metabolic control, to lessen complications and to improve patient's quality of life.

Key words: Type 2 Diabetes Mellitus, practice guidelines, health care.

Fecha recepción: 15/11/11 Fecha aceptación: 16/11/12

\section{INTRODUCCIÓN}

La Diabetes Mellitus es, a nivel mundial, un problema de salud pública de alto costo para las personas, las familias y los países. Actualmente afecta al 7\% de la población adulta mundial (1). En Latinoamérica, la prevalencia de la diabetes mellitus tipo 2 (DM2) en menores de 30 años es menor al 5\% mientras que después de los 60 años sube a más del $20 \%$ (2).

La Federación Internacional de Diabetes indica que las personas de países de renta media-baja están soportando el peso de este problema de salud y que está afectando más de lo que previamente se creía a personas en edad laboral; además predice que al 2030 la cifra de personas con esta enfermedad excederá los 435 millones y que la diabetes costará a la economía mundial más de 490 billones de US\$ (1). Como ejemplo, la Organización Mundial de la Salud (OMS) calcula que en el 2006-2015 China dejará de percibir ingresos nacionales de US\$ 558.000 millones a causa de las cardiopatías, los accidentes cerebrovasculares (ACV) y la diabetes (3).

La diabetes es la causa principal de ceguera, disfunción renal, ataques cardíacos, infartos y amputación (1).

En Chile, debido a los cambios en la estructura demográfica de la población, la prevalencia de la diabetes ha aumentado desde el 2003 al 2010 en un 3.1\% (en varones un $2.3 \%$ y en mujeres un $3,9 \%)(4,5)$. La prevalencia en el tramo de 65 años y más es de un $25,8 \%$ (5) y significativamente más alta en el nivel socioeconómico bajo que en los niveles más altos: $4,3 \%$ y $5,3 \%$ en los niveles $\mathrm{D}$ y $\mathrm{E}$ respectivamente comparado con el $1,7 \%$ en el grupo $\mathrm{ABC1}^{2}$ (5).

La enfermedad en sí, su manejo y la alta prevalencia de complicaciones $(5,6)$ impac-

${ }^{2} \mathrm{ABC1}$ : Clase alta, media alta; C2: Clase media media; C3: Clase media baja; D: Pobreza; E: Extrema pobreza.

Instituto Nacional de Estadísticas. Estratificación Socioeconómica en Encuestas de Hogares; 2012. 
ta en muchos aspectos de la vida cotidiana de los pacientes, como por ejemplo en el trabajo, las relaciones personales, sociales y familiares, en el bienestar físico y psicológico, entre otros. Este impacto se expresa como "calidad de vida" (7). Las complicaciones, debido principalmente a un mal control metabólico, son las que tienen mayor impacto en ella, como la vasculopatía periférica, la retinopatía, la cardiopatía isquémica y la nefropatía, entre otras $(6,8)$. Diversos autores plantean que esta enfermedad produce un deterioro gradual y progresivo de la calidad de vida en las personas según el avance de la enfermedad $(9,10)$. Las áreas que se ven más afectadas son la capacidad funcional y la sensación de bienestar; también puede haber un impacto emocional y social importante que puede causar alteraciones psicológicas graves. Esto se ve acentuado por las complicaciones y enfermedades asociadas (5), como también debido a la dificultad de seguir un tratamiento farmacológico y no farmacológico de por vida $(5,11)$. Los grupos de diabéticos más afectados en su calidad de vida son las mujeres, los obesos, los ancianos y las personas que viven solas con bajo ingreso económico $(12,13)$.

Las políticas públicas de salud en nuestro país abordan esta problemática a través de distintas estrategias: dos de ellas son la incorporación de esta enfermedad a las Garantías Explícitas en Salud (GES) (14) y la implementación del Programa Cardiovascular con sus protocolos que tratan de dar respuesta al problema de la diabetes estableciendo las recomendaciones para la atención primaria a través de la Guía Clínica Diabetes Mellitus tipo 2 (15). Sin embargo, nuestro país no ha logrado la meta de frenar el ascenso en la mortalidad por diabetes, planteada en los objetivos sanitarios 2000-2010 (16).

La falta de un manejo efectivo de estos pacientes, según el Ministerio de Salud (MIN$\mathrm{SAL}$ ), y la baja adherencia a las acciones recomendadas, según otros estudios $(15,17)$, queda de manifiesto en: la baja proporción de pacientes bien controlados, el bajo porcentaje de pacientes diabéticos controlados en el nivel primario de atención con buen control metabólico (36\%) (15), el creciente número de pacientes diabéticos que ingresa a diálisis y la alta frecuencia de pacientes con pie diabético que deben ser amputados, entre otras complicaciones prevenibles.

Las recomendaciones del MINSAL se sustentan en el Chronic Care Model; este modelo identifica diversos componentes críticos de los sistemas de salud asociados a mejores resultados, entre ellos el trabajo en equipo al servicio de las necesidades de los pacientes (5). El trabajo en equipo en la práctica de la atención primaria muestra la efectividad en el mantenimiento del estado de salud, especialmente en las personas mayores con enfermedades crónicas (18), ya que se potencia el logro de objetivos a través de la sumatoria de capacidades individuales y especificidad de cada profesional.

Las enfermeras tienen un papel fundamental en la atención primaria y una gran influencia en el equipo de salud (19). Del trabajo conjunto de enfermeras y médicos depende en gran medida el logro de los objetivos que el MINSAL se ha propuesto a través de la creación del Programa Cardiovascular, al cual se incorporan los pacientes diabéticos.

Respecto a las enfermeras, en específico, el MINSAL les asigna un rol importante en las prestaciones incorporadas al Programa Cardiovascular. Además de las actividades de carácter asistencial, la enfermera tiene un papel preponderante en educación. Es reconocida la importancia que tiene la educación para involucrar al paciente diabético en su cuidado y fomentar su adherencia al tratamiento. Esta educación es parte de las actividades que se contemplan en el Programa Cardiovascular.

Dada la significativa participación de enfermería en la implementación y en los resultados del Programa Cardiovascular, se hace relevante medir cómo se está llevando 
dicho programa y cuál es el efecto que está teniendo en la salud de los pacientes diabéticos. Esto le permite a la enfermera y al equipo de salud validar el quehacer de una manera científica y corregir oportunamente el plan de trabajo en aquellas áreas en que no se observan los resultados esperados. De ahí que el presente estudio tenga como propósito conocer el nivel de cumplimiento, por parte del equipo de salud, de las recomendaciones ministeriales y de la eficacia de éstas en la salud de la población

Preguntas de investigación: en los pacientes diabéticos tipo 2 controlados en un Centro de Salud Familiar (CESFAM) del Servicio de Salud Metropolitana Oriente (SSMO) de Santiago ¿Cuál es el efecto en la condición de salud de la población, de las atenciones entregadas por los profesionales de salud de acuerdo a las recomendaciones ministeriales? ¿Qué variables se comportan como factor de riesgo o protector de la condición de salud? Objetivos: Caracterizar a la población estudiada según variables sociodemográficas, tiempo de diagnóstico y control de la diabetes. Identificar la condición de salud (CS) de los pacientes diabéticos tipo 2 en estudio a través de seis aspectos. Medir el cumplimiento de las atenciones de salud según la recomendación ministerial. Identificar variables asociadas a la condición de salud.

Variables principales: condición de salud medida a través de: compensación de la diabetes (HbAlc < a 7\%), patologías crónicas agregadas según diagnóstico médico, se consideran hipertensión arterial, dislipidemia, otras. Grado de autovalencia según última encuesta de detección de riesgo de pérdida de funcionalidad en el adulto mayor (EFAM), complicaciones de la diabetes según diagnóstico médico; se consideran nefropatía diabética incipiente, insuficiencia renal crónica, neuropatía diabética, amputación, retinopatía diabética, ceguera, infarto agudo del miocardio, accidente vascular encefálico y riesgo de pie diabético: categorizado en riesgo máximo, alto y moderado de acuerdo a "Norma Técnica Manejo Integral del Pie Diabético” (20), estado nutricional según diagnóstico nutricional en último control, presión arterial medida al momento del estudio.

Atenciones de salud según recomendación ministerial: el cumplimiento de las recomendaciones ministeriales se establece comparando los resultados obtenidos con las indicaciones de atenciones sugeridas en la Guía clínica de Diabetes Mellitus tipo 2 del Ministerio de Salud (15). Se consideran las siguientes atenciones: controles profesionales anuales por paciente (a lo menos 5); evaluación de pie diabético, al menos una vez al año, según instrumento del MINSAL de valoración de riesgo de ulceración y amputación del pie; exámenes de control (batería de exámenes de sangre, orina, electrocardiograma y fondo de ojo) a lo menos una vez al año; indicación de régimen por una nutricionista en el último control; educación por parte del médico, de la nutricionista y de la enfermera en el último control en cuanto a: enfermedad, actividad física, alimentación y cuidado de los pies.

\section{MATERIAL Y MÉTODO}

Estudio de prevalencia puntual a abril 2010. Población de 1.804 pacientes diabéticos tipo 2, controlados en el Programa de Salud Cardiovascular a abril del 2010, en un Centro de Salud Familiar (CESFAM) de la comuna de Macul. Criterios de inclusión: paciente diabético tipo 2, de 20 años o más, con a lo menos un año en control. Criterios de exclusión: pacientes con diagnóstico de algún tipo de demencia, postrados e inasistentes a los controles por más de un año. La población a estudiar es de 1.100 pacientes. Se extrajo una muestra probabilística de 340 pacientes por aleatorización simple, a través de programa computacional Excel, asignando a las estimaciones una confianza del 95\% y consi- 
derando una merma del 10\%. Los pacientes incluidos en la muestra aceptaron participar en el estudio a través de consentimiento informado. La fuente de información fue la ficha clínica para las variables condición de salud y atenciones de salud entregadas según recomendación ministerial; para las variables sociodemográficas, familiares y tiempo de enfermedad, la fuente de información fue el paciente. Para recoger la información se diseñó un instrumento que consta de dos partes: la primera recoge la información de la ficha y exámenes y la segunda es una encuesta al paciente con preguntas cerradas, la cual fue previamente revisada por expertos y a través de una prueba piloto en 20 pacientes que no participaron en el estudio. La recolección de datos se realizó entre mayo y agosto de 2010; en la recolección participaron las propias investigadoras, algunas enfermeras del CESFAM e internas de enfermería de la Universidad de los Andes previamente entrenadas. Se citó a los pacientes al CESFAM; de no cumplir con la citación, se realizó una visita domiciliaria. El análisis de datos y evaluación de resultados se realizó a través de los programas computacionales Stata 5.0; Epi Info 3.5.1 y Excel 2003, con estadística descriptiva y medidas de resumen. Para la asociación de variables se utilizó $\mathrm{Chi}^{2}$ y Odds Ratios con sus intervalos correspondientes con un $95 \%$ de confianza. El proyecto de investigación contó con la aprobación del Comité de Ética del SSMO y del Comité de Ética de la Universidad de los Andes.

\section{RESULTADOS}

La población estudiada tiene en promedio 66.5 años; el 72\% corresponde a adultos mayores de 60 años. Predomina el sexo femenino con un $61.2 \%$. El promedio de años de estudio es de 8.7 años (DS 4.37); un 47.8\% de las mujeres tiene menos de 8 años de estudio a diferencia de los hombres, entre quienes sólo un $26.5 \%$ tiene ese nivel de escolaridad ( $\mathrm{p}=0.000)$. Casi la mitad de la población $(47.8 \%)$ tiene un ingreso familiar mensual menor a $\$ 150.000$. Un $42.6 \%$ tiene diagnosticada la enfermedad hace más de 10 años pero sólo un $21 \%$ se la controla hace más de 10 años.

La condición de salud se describe en la Tabla 1. Un 43.5\% está descompensado de la diabetes; no hubo diferencia estadísticamente significativa según sexo.

Tabla 1. Condición de salud de los pacientes en estudio $(n=340)$

\begin{tabular}{|lrc|}
\hline Condición de salud & $\mathrm{N}^{\circ}$ & $\%$ \\
\hline Descompensación de la diabetes & 148 & 43.5 \\
Patología agregada & 333 & 97.9 \\
Complicaciones de la diabetes & 85 & 25.0 \\
Descompensación presión arterial & 198 & 58.2 \\
Estado nutricional alterado por exceso & 242 & 71.2 \\
Autovalencia adultos mayores $(\mathrm{n}=199)$ & 46 & 23.1 \\
\hline
\end{tabular}

De los pacientes que tienen patología agregada (333) 97\% tienen diagnóstico de hipertensión arterial y un $67 \%$ de dislipidemia. De los pacientes que presentan com- plicaciones (85), las más frecuentes son retinopatía diabética (41\%), infarto agudo del miocardio $(33 \%)$ e insuficiencia renal crónica $(17.6 \%)$. De los 199 pacientes de 65 años 
o más, 9.5\% son dependientes, no existiendo diferencia estadísticamente significativa según sexo $(\mathrm{p}=0.882)$.
Las variables sociodemográficas asociadas a distintos aspectos de la condición de salud se presentan en la Tabla 2.

Tabla 2. Relación entre condición de salud/variables sociodemográficas de los pacientes en estudio.

\begin{tabular}{|lcccc|}
\hline & $\mathbf{C h i}^{2}$ & $\mathbf{p}$ & OR & IC \\
\hline Presión arterial/edad (> 65 años) & 17.49 & 0.000 & 4.56 & $2.01-10.43$ \\
Amputacion/Sexo (hombres) & 8.8 & 0.003 & 7.52 & $1.49-51.29$ \\
Alteración nutricional por exceso/Edad (<65 años) & 14.78 & 0.000 & 3.5 & $1.94-6.45$ \\
Alteración nutricional por exceso/Sexo (mujeres) & 8.62 & 0.003 & 2.3 & $1.23-3.37$ \\
Alteración nutricional por exceso/Actividad (trabajador) & 8.09 & 0.004 & 2.5 & $1.4-4.1$ \\
\hline
\end{tabular}

El Gráfico 1 muestra el grado de cumplimiento de las recomendaciones ministeriales estudiadas. El promedio de controles por paciente en el último año es de 4.1, con un mínimo de 0 y un máximo de 13 . El CESFAM adapta la recomendación ministerial de realizar a lo menos 5 controles anuales por profesionales a los pacientes diabéticos tipo 2 de la siguiente manera: a los pacientes compensados les realiza a lo menos 3 controles profesionales anuales y a los pacientes descompensados a lo menos 4 controles.

Gráfico 1. Cumplimiento de recomendación ministerial de atenciones prestadas por profesionales a los pacientes en estudio.

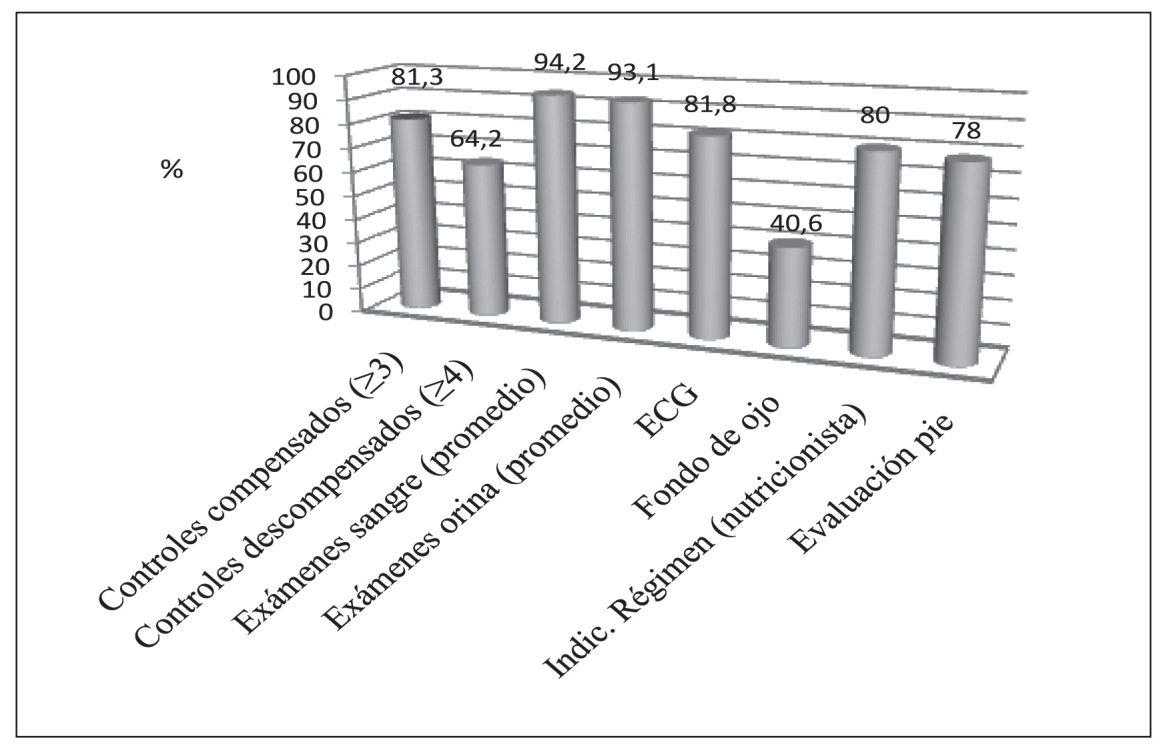

Los pacientes compensados, de acuerdo a los distintos aspectos de la condición de salud, tienen un porcentaje mayor de cumpli- miento de asistencia a los controles que los descompensados $(81,3 \%$ y $64,2 \%$ respectivamente). En la Tabla 3 se detalla el cumpli- 
Tabla 3. Número de pacientes citados y controlados por profesional el último año (n=340).

\begin{tabular}{|lcccccc|}
\hline & \multicolumn{3}{c}{ Pacientes citados } & \multicolumn{3}{c|}{ Pacientes controlados } \\
\hline Profesional & $\mathrm{N}$ & $\%$ & Promedio & $\mathrm{N}$ & $\%$ & Promedio \\
\hline Enfermera & 306 & 90 & 1.8 & 296 & 87.1 & 1.3 \\
Médico & 332 & 97.6 & 2.4 & 325 & 95.6 & 1.9 \\
Nutricionista & 267 & 78.5 & 1.3 & 234 & 68.8 & 1.0 \\
\hline
\end{tabular}

miento de la normativa ministerial en relación a citaciones y controles de los pacientes, según profesionales.

De los 340 pacientes estudiados, a 29 (8.5\%) no se les tomó HbAcl el último año y a un $17.1 \%$ no se le realizó microalbuminuria.
El registro de la educación indicada por los profesionales se detalla en Tabla 4. La enfermera es la profesional con mayor número de registros en todos los aspectos.

$\mathrm{Al}$ asociar componentes de condición de salud con los de cumplimiento de recomendación ministerial, no hubo asociación esta-

Tabla 4. Porcentaje de pacientes con registro de educación en la ficha, según profesional.

\begin{tabular}{|c|c|c|c|}
\hline Registro Educación & $\%$ médico & $\%$ nutricionista & $\%$ enfermera \\
\hline Cuidado de los pies & 2.4 & 0.6 & 55.6 \\
\hline Alimentación & 48.5 & 68.8 & 82.9 \\
\hline Actividad física & 19.7 & 11.8 & 66.8 \\
\hline Sobre su enfermedad & 6.5 & 3.8 & 20 \\
\hline
\end{tabular}

dísticamente significativa entre el número de controles totales realizados a los pacientes en el último año y la compensación de la diabetes $(\mathrm{p}=0.066)$. Al categorizar la variable número de controles en menos de 5 y más de 5 , se observa una asociación estadísticamente significativa con la compensación de la diabetes ( $\mathrm{p}=0.009)$, comportándose como factor protector, IC (0.62-0.95). No se encontró asociación estadísticamente significativa con el resto de las variables

\section{DISCUSIÓN Y CONCLUSIÓN}

Las características generales de la población en estudio, con predominio de mujeres, de adultos mayores y de personas de baja esco- laridad, son coincidentes con otros estudios (21-23). Sin embargo, los estudios citados tienen una población con un nivel de escolaridad aún más bajo que el de nuestra población.

Los años en control de la diabetes son menores que los años de diagnóstico; esto se puede deber a la dificultad de los pacientes para asumir la enfermedad crónica o a que la adherencia de los pacientes al control mejora cuando aparecen signos y síntomas. El 42,6\% de los pacientes padece la enfermedad hace más de 10 años, comparado con estudios en que sólo un 22,2\% (7) o un $31,4 \%$ (21) de los pacientes han sufrido la enfermedad por ese número de años. Una mejora en la percepción del paciente sobre las consecuencias que pueden derivar de la progresión de su enfermedad permitiría un 
mejor control de ésta (24).

Los componentes de la condición de salud, en general, están por sobre lo esperado. Un 56.5\% de los pacientes está compensado; este porcentaje sobrepasa la meta sanitaria (40\%) y es mayor a lo encontrado en la Encuesta Nacional de Salud 2009-2010 (36\%). En estudios internacionales se muestran cifras sobre $70 \%$ de descompensación $(21,23)$. Si bien estos resultados estarían dentro de lo esperado, es importante tener en cuenta que un $43.5 \%$ tiene un mal control metabólico, lo que se traduce en un mayor riesgo de complicaciones.

Coincidiendo con otros estudios $(7,13$, 21), se encontró una alta frecuencia de HTA, obesidad y dislipidemia, enfermedades propias del síndrome metabólico presente en muchos de los pacientes con DM2. La alteración nutricional por exceso que presentan los pacientes $(71.2 \%)$ coincide con los resultados de diferentes autores $(7,12,21,23)$. Las enfermedades asociadas deterioran en forma significativa la capacidad funcional y la sensación de bienestar de las personas (11). Estos estudios corroboran que, a medida que los pacientes diabéticos presentan sobrepeso u obesidad, se afecta directamente su capacidad física, su bienestar psicológico y, por ende, su calidad de vida.

Se ha comprobado que el factor que condiciona en mayor medida el deterioro de la calidad de vida, relacionada con la condición de salud, es la existencia de complicaciones (11). En el presente estudio, el 25\% de los pacientes tiene alguna complicación; las complicaciones más frecuentes coinciden con los resultados presentados por otros autores (6, 12). Los pacientes con nefropatía incipiente constituyen un $11.8 \%$, lo que indicaría una baja pesquisa en relación a lo esperado a nivel nacional $(30 \%)$. La amputación, siendo una complicación prevenible, se da en un porcentaje no despreciable de pacientes. Habría que investigar qué factores influyen en que los hombres tengan más riesgo de esta complicación. A este respecto, cabe preguntarse si los hombres tienen menor conciencia de autocuidado.

En relación al cumplimiento de la recomendación, se observa que los pacientes tienen atención por un equipo multidisciplinario de profesionales, entre ellos médicos, enfermeras y nutricionistas. Si bien en ningún paciente se cumplen en un $100 \%$ las recomendaciones ministeriales, el cumplimiento de las metas del Programa de Salud cardiovascular del CESFAM está por sobre las metas ministeriales. Sin embargo podrían mejorarse los indicadores. Acortar citas para el seguimiento de los pacientes mejora los resultados del proceso (25), lo que coincide con la recomendación ministerial y los resultados de este estudio en cuanto a que la asistencia a 5 o más controles es un factor favorecedor de la compensación de la diabetes. Cabe hacer notar que a un $8.5 \%$ de los pacientes no se les realizó hemoglobina glicosilada durante el último año y a un $17.1 \%$ no se le realizó microalbuminuria, aspectos importantes de tener en cuenta ya que la hemoglobina glicosilada es el indicador estándar de la compensación de la diabetes y la microalbuminuria es un buen predictor de daño renal.

Tanto el cumplimiento de la normativa de solicitud como la realización del fondo de ojo es de un 50\%; esto influye en la pesquisa temprana de retinopatía diabética siendo ésta, en el presente estudio, la principal complicación de la diabetes. Sería importante tener en cuenta la recomendación del estudio de Vijan (26), que plantea que en los pacientes de alto riesgo (jóvenes y con niveles altos de HbAlc) el intervalo más coste-efectivo de realización de fondo de ojo sería un año y en los casos de bajo riesgo (mayores y con buenos niveles de HbA1c) sería 3 años.

El bajo cumplimiento de la normativa de indicación de régimen alimenticio por la nutricionista en el último control y de los resul- 
tados en cuanto a la educación entregada por los profesionales podría deberse a subregistro. Es importante ayudar a los profesionales a tomar conciencia sobre la importancia de los registros, ya que sin ellos es imposible acercarse a la realidad de las acciones realizadas para luego evaluar su impacto en la salud de la población.

Se debe considerar la educación como elemento fundamental para la prevención, lo que implica que debe ser realizada y reforzada por todo el equipo de salud para así dar a conocer a los pacientes la importancia de los cuidados y motivarlos a practicarlos. En este sentido, si bien se espera que el equipo de salud invierta tiempo y esfuerzo en informar y educar a los pacientes, esto no se ve reflejado en los registros. Los resultados de este estudio han permitido conocer con más detalle el nivel de cumplimiento de las recomendaciones ministeriales en cuanto a las atenciones entregadas por los profesionales y el impacto de ellas a través de la condición de salud de los pacientes, donde se observa que el porcentaje de pacientes compensados metabólicamente supera la meta ministerial (>40\%), sin embargo, considerando los riesgos asociados a la descompensación de la diabetes, sería recomendable incrementar el porcentaje de pacientes compensados. La adaptación que hace el CESFAM de la recomendación ministerial en lo referente al número de controles profesionales al año no favorece la condición de salud de los pacientes. Aumentar el número de controles/profesionales/año a 5 o más, como recomienda el MINSAL, mejora el estado de compensación de la diabetes en los pacientes. Características sociodemográficas se asocian a condición de salud, entre ellas el sexo. Sería interesante replicar este estudio en los otros centros de la comuna, como también en establecimientos de diferentes características de la región o del país para poder extrapolar los resultados a un mayor universo.

\section{REFERENCIAS}

1. Federación Internacional de Diabetes (FID). Las últimas cifras sobre diabetes muestran un panorama desalentador [Internet]. Montreal: Federación Internacional de Diabetes; 2009 [citado 2 abril 2010] Disponible en: http://www.idf. org/webdata/docs/PR_IDFDiabetesAtlas191009_ES.pdf

2. Asociación Latinoamericana de Diabetes (ALAD). Guías ALAD de diagnóstico, control y tratamiento de Diabetes Mellitus tipo 2 [Internet]. Washington, D.C: Asociación Latinoamericana de Diabetes; 2008 [citado 3 marzo 2010] Disponible en: http://revistaalad.com.ar/guias/ GuiasALAD_DMTipo2_v3.pdf

3. Organización Mundial de la Salud (OMS). Diabetes. Nota descriptiva No 312 [Internet] Ginebra: WHO Media Centre; enero 2011 [citado 12 marzo 2011] Disponible en: http://www.who.int/mediacentre/ factsheets/fs312/es/index.html

4. MINSAL. Departamento Epidemiología. Encuesta nacional de salud [Internet]. Chile: Ministerio de Salud; 2003 [citado 12 abril 2010]. Disponible en: http://epi. minsal.cl/.

5. Ministerio de Salud. Departamento de Epidemiología. Encuesta Nacional de Salud [Internet]. Chile: Ministerio de Salud; 2009-2010 [citado 10 abril 2010]. Disponible en: http://epi.minsal.cl/.

6. Zafra JA, Méndez JC, Novalbos JP. Complicaciones crónicas en los pacientes con diabetes mellitus tipo 2 atendidos en un centro de salud. Aten Primaria. 2000; 25(8): 29-43.

7. Vicente B, Zerquera G, Peraza D, Castañeda E, Irizar J, Valladares T. Calidad de vida en el paciente diabético. MediSur [Internet]. 2008 [citado 15 marzo 2010]; 6(3): 22-8. Disponible en: http://www. medisur.sld.cu/index.php/medisur/issue/view/30. 
8. Base de Datos Cochrane Intervenciones para mejorar el cumplimiento de las recomendaciones de tratamiento en personas con diabetes mellitus tipo 2 [Internet]. Oxford (UK); 2008 [citado 17 de agosto de 2010]. Disponible en: http:// www.update-software.corn/cochrane

9. Hervás A, Zabaleta A, De Miguel G, Beldarrain O, Díez J. Calidad de vida relacionada con la salud en pacientes con diabetes mellitus tipo 2. An Sist Sanit Navar. 2007; 30(1): 45-52.

10. De los Ríos JL, Sánchez JJ, Barrios P, Avila TL. Quality of life in patients with diabetic nephropathy. Invest Educ Enferm. 2005; 23(1): 30-43.

11. Mena FJ, Martín JC, Simal F, Bellido J, Carretero JL. Diabetes Mellitus tipo 2 y calidad de vida relacionada con la salud: resultados del Estudio Hortega. An Med Interna. 2006; 23(8): 357-60.

12. Quirantes A, López L, Curbelo V, Montano JA, Machado $P$ et al. La calidad de la vida del paciente diabético. Rev Cubana Med Gen Integr. 2000; 16(1): 50-6.

13. Base de Datos Cochrane Entrenamiento grupal de estrategias de autocuidado en personas con diabetes mellitus tipo 2 [Internet]. Oxford (UK); 2008 [actualizado mayo 2008, citado 4 mayo 2010]. Disponible en: http://www.update-software. corn/cochrane/.

14. Superintendencia de Salud, Gobierno de Chile. Decreto Supremo No 44 Aprueba Garantías explícitas en salud del Régimen General de Garantías en Salud. Diario Oficial (Fiscalía). 31 de enero 2007.

15. Ministerio de Salud. Guía Clínica Diabetes Mellitus tipo 2 [Internet]. Santiago, Chile: MINSAL; 2009 [citado 13 abril 2010] Disponible en: http://www. redcronicas.cl/index.php?option $=\mathrm{com}_{-}$ docman\&Itemid $=114$.

16. Ministerio de Salud. Objetivos Sanitarios de la década 2000-2010. Evaluación de final de período [Internet]. Santiago, Chi- le: Ministerio de Salud, Departamento de Epidemiología; 2011 [citado 13 abril 2010]. 88-9 p. Disponible en: http://epi. minsal.cl

17. McGlynn EA, Asch SM, Adams J, Keesey J, Hicks J et al. The Quality of Health Care Delivered to Adults in the United States. N Engl J Med. 2003; 348: 2635-645.

18. Sommers LS, Marton KI, Barbaccia JC, Randolph J. Physician, Nurse, and Social Worker Collaboration in Primary Care for Chronically III Seniors. Arch Intern Med. 2000; 160(12):1825-833.

19. Harrison S, Dowswell G, Wright J. Practice nurses and clinical guidelines in a changing primary care context: an empirical study. J Adv Nurs. 2002; 39: 299-307.

20. Ministerio de Salud. Guía Clínica Diabetes Mellitus tipo 2. Norma Técnica Manejo Integral del Pie Diabético [Internet]. Santiago, Chile: MINSAL; 2006 [citado 13 abril 2010]. Disponible en: http:// www.redsalud.gov.cl/archivos/guiasges/ diabetesGes.pdf

21. López-Carmona JM, Rodríguez-Moctezuma R. Adaptación y validación del instrumento de calidad de vida Diabetes 39 en pacientes mexicanos con diabetes mellitus tipo 2. Salud Publ Mex. 2006; 48(3): 200-8.

22. Árcega-Domínguez A, Lara-Muñoz C, Ponce-de-León-Rosales S. Factores relacionados con la percepción subjetiva de la calidad de vida de pacientes con diabetes. Rev Invest Clin. 2005; 57 (5): 676-84.

23. Cárdenas V, Pedraza C, Lerma R. Calidad de vida del paciente con diabetes mellitus tipo 2. Ciencia UANL. 2005; 8(3):351-57.

24. Mata M, Roset M, Badia X, Antoñanzas F, Ragel J. Impacto de la diabetes mellitus tipo 2 en la calidad de vida de los pacientes tratados en las consultas de atención primaria en España. Aten Primaria. 2003; 31(8): 493-9.

25. Base de Datos Cochrane Intervenciones para mejorar el tratamiento de la diabe- 
tes mellitus en el ámbito de la atención primaria, pacientes ambulatorios y la comunidad [Internet]. Oxford (UK); 2008 [actualizado mayo 2008, citado 17 de agosto de 2010]. Disponible en: http:// www.update-software.com/cochrane/.
26. Vijan S, Hofer TP, Hayward RA. Costutility analysis of screening intervals for diabetic retinopathy in patients with type 2 diabetes mellitus. JAMA. 2000; 283(7): 889-96. 